\title{
First report of Toxoplasma gondii infection in market-sold adult chickens, ducks and pigeons in northwest China
}

Wei Cong ${ }^{1,2}$, Si-Yang Huang ${ }^{1}$, Dong-Hui Zhou ${ }^{1}$, Min-Jun Xu', Song-Ming Wu' ${ }^{1}$, Chao Yan ${ }^{3}$, Quan Zhao², Hui-Qun Song ${ }^{1}$ and Xing-Quan Zhu ${ }^{1,2,4^{*}}$

\begin{abstract}
Background: Toxoplasma gondii infection is a global concern, affecting a wide range of warm-blooded animals and humans worldwide, including poultry. Domestic and companion birds are considered to play an important role in the transmission of T. gondii to humans and other animals. However, little information on T. gondii infection in domestic birds in Lanzhou, northwest China was available. Therefore, this study was performed to determine the seroprevalence of T. gondii infection in domestic birds in Lanzhou, northwest China.

Methods: In the present study, the seroprevalence of T. gondii antibodies in 413 (305 caged and 108 free-range) adult chickens, 334 (111 caged and 223 free-range) adult ducks and 312 adult pigeons in Lanzhou, northwest China, were examined using the modified agglutination test (MAT).

Results: 30 (7.26\%) chickens, 38 (11.38\%) ducks and 37 (11.86\%) pigeons were found to be positive for T. gondii antibodies at the cut-off of 1:5. The prevalences in caged and free-range chickens were $6.23 \%$ and $10.19 \%$ respectively, however, statistical analysis showed that the difference was not significant $(P>0.05)$. The seroprevalences in caged and free-range ducks were $6.31 \%$ and $13.90 \%$ respectively, but the difference was not statistically significant $(P>0.05)$.

Conclusions: The results of the present survey indicated the presence of T. gondii infection in adult chickens, ducks and pigeons sold for meat in poultry markets in Lanzhou, northwest China, which poses a potential risk for T. gondii infection in humans and other animals in this region. This is the first seroprevalence study of $T$. gondii infection in domestic birds in this region.
\end{abstract}

\section{Background}

Toxoplasma gondii is an important intracellular protozoan parasite, widely prevalent in humans and animals, including poultry throughout the world [1-3]. T. gondii infection is generally transmitted either congenitally, or via ingestion of undercooked or raw meat from infected animals, or ingestion of food or water contaminated with oocysts excreted by infected felids [1,2,4]. Free-range

\footnotetext{
*Correspondence: xingquanzhu1@hotmail.com

'State Key Laboratory of Veterinary Etiological Biology, Key Laboratory of Veterinary Parasitology of Gansu Province, Lanzhou Veterinary Research Institute, Chinese Academy of Agricultural Sciences, Lanzhou, Gansu Province 730046, People's Republic of China

${ }^{2}$ College of Animal Science and Technology, Jilin Agriculture University,

Changchun, Jilin Province 130118, People's Republic of China

Full list of author information is available at the end of the article
}

(FR) chickens, an important intermediate host, are considered one of the best indicators for soil contamination with T. gondii oocysts because of their feeding style [2]. Low numbers of exposed poultry develop clinical symptoms, such as encephalitis, chorioretinitis and neuritis, however, poultry meat is an important part of cuisine, consumed widely all over the world, and consumption of uncooked poultry meat or not properly cooked poultry meat is a risk factor for $T$. gondii infection in humans or other animals $[1,2]$.

In recent years, seroprevalence studies of $T$. gondii in chickens, ducks and pigeons have been conducted extensively in various parts of the world [1,2,5-7], there have been some surveys in mainland China [8-10]. However, little is known about the prevalence of $T$. gondii in

\section{Biomed Central}


chickens, ducks and pigeons in northwest China. Here, we report T. gondii seroprevalence in domestic birds in Lanzhou, northwest China for the first time.

\section{Methods}

\section{The study area}

The survey was conducted in Lanzhou City $\left(35^{\circ} 5^{\prime} \sim 38^{\circ}\right.$ N, $102^{\circ} 30^{\prime} \sim 104^{\circ} 30^{\prime}$ E), the Capital of Gansu Province, northwest China. Lanzhou is situated in the geometric center of China, covering an area of approximately 13,000 square kilometers, with an average altitude of 1,500 meters. The climate of this city is typically temperate and monsoonal continental, with an average annual temperature of $9.3{ }^{\circ} \mathrm{C}$, and an annual precipitation of $360 \mathrm{~mm}$.

\section{Blood samples}

A total of 413 blood samples from adult chickens, 334 blood samples from adult ducks and 312 blood samples from adult pigeons were collected from animals slaughtered and sold for meat in four poultry markets (poultry market A, B, C, D) in Lanzhou, northwest China between April and November 2011. Blood samples were transported to the laboratory in Lanzhou Veterinary Research Institute, Chinese Academy of Agricultural Sciences, Lanzhou, Gansu Province, China, kept at room temperature for $2 \mathrm{hr}$, centrifuged at $3,000 \mathrm{~g}$ for $10 \mathrm{~min}$, then clear serum was separated. The serum samples obtained were stored at $-20{ }^{\circ} \mathrm{C}$ until further analyzed. Owners of poultry were asked for information of animal husbandry practices.

\section{Serological examination}

Antibodies to T. gondii were determined in chicken, duck and pigeon sera by the modified agglutination test (MAT) as described previously $[2,8,11,12]$. In brief, sera were added to the " $U$ " bottom of 96 well microtiter plates, and diluted two-fold starting from 1:5 to 1:160. Bird sera with MAT titers of 1:5 or higher were considered positive for $T$. gondii antibodies based on previous studies [2,8,13-15], those sera with doubtful reactions were re-tested, and positive and negative controls were included in each test.

\section{Statistical analyses}

Differences in the seroprevalence of $T$. gondii-infected chickens and ducks and between free-range and caged groups were analyzed using a Chi square test in SPSS for Windows (Release 18.0 standard version, SPSS Inc., Chicago, Illinois). The differences were considered statistically significant when $P<0.05$.

\section{Results}

One hundred and five (9.92\%) out of 1059 serum samples were assayed positive for $T$. gondii by MAT (Table 1). Of these, a total of $30(7.26 \%)$ out of 413 chickens were seropositive and antibody titers were 1:5 in 20, 1:10 in seven, 1:20 in one, 1:40 in one and 1:80 in one chicken (Table 1). As shown in Table 2, the seroprevalence varied in different poultry markets, ranging from $1.73 \%$ to $10.19 \%$, and the seroprevalence in caged and free-range chickens was $6.23 \%$ and $10.19 \%$, respectively.

Antibodies to T. gondii were found in 38 (11.38\%) out of 334 ducks, and antibody titers were 1:5 in 33 and 1:10 in five ducks (Table 1). T. gondii seroprevalences in ducks came from four different poultry markets ranging from $0 \%$ to $18.07 \%$ (Table 2). The investigation revealed that the prevalence in caged and free-range ducks was $17.61 \%$ and $12.72 \%$, respectively.

T. gondii antibodies were detected in 37 (11.86\%) of 312 examined pigeons with antibody titers of 1:5 in 35 and 1:10 in two (Table 1), and the seroprevalences of T. gondii in four different poultry markets ranged from $2.52 \%$ to $22.95 \%$ (Table 2 ).

\section{Discussion}

In this investigation, seroprevalence of $T$. gondii infection in chickens was $7.26 \%$, which was lower than that observed in other countries [2], and also lower than that

Table 1 Seroprevalence of Toxoplasma gondii infection in chickens, ducks and pigeons in Lanzhou, northwest China by modified agglutination test (MAT)

\begin{tabular}{|c|c|c|c|c|c|c|c|c|}
\hline \multirow[t]{2}{*}{ Host } & \multicolumn{5}{|c|}{ No. of sera with MAT titers of: } & \multirow{2}{*}{$\begin{array}{c}\text { No. } \\
\text { positive }\end{array}$} & \multirow{2}{*}{$\begin{array}{c}\text { No. } \\
\text { tested }\end{array}$} & \multirow{2}{*}{$\begin{array}{c}\text { Prevalence } \\
(\%)\end{array}$} \\
\hline & $1: 5$ & $1: 10$ & $1: 20$ & $1: 40$ & $1: 80$ & & & \\
\hline Caged chickens & 13 & 4 & 0 & 1 & 1 & 19 & 305 & 6.23 \\
\hline FR chickens & 7 & 3 & 1 & 0 & 0 & 11 & 108 & 10.19 \\
\hline Caged ducks & 7 & 0 & 0 & 0 & 0 & 7 & 111 & 6.31 \\
\hline FR ducks & 26 & 5 & 0 & 0 & 0 & 31 & 223 & 13.90 \\
\hline Pigeons & 35 & 2 & 0 & 0 & 0 & 37 & 312 & 11.86 \\
\hline Total & 88 & 14 & 1 & 1 & 1 & 105 & 1059 & 9.92 \\
\hline
\end{tabular}

FR: Free-range. 
Table 2 Seroprevalence of Toxoplasma gondii infection in chickens, ducks and pigeons in different poultry markets in Lanzhou, northwest China by modified agglutination test (MAT)

\begin{tabular}{cllll}
\hline Host & Poultry market & No. tested & No. positive & Prevalence (\%) \\
\hline Chicken & $\mathrm{A}$ & 97 & 9 & 9.28 \\
\cline { 2 - 5 } & $\mathrm{B}$ & 113 & 2 & 1.77 \\
\cline { 2 - 5 } & $\mathrm{C}$ & 108 & 11 & 10.19 \\
\cline { 2 - 5 } & $\mathrm{D}$ & 95 & 8 & 8.42 \\
\hline Duck & $\mathrm{A}$ & 63 & 4 & 6.35 \\
\cline { 2 - 5 } & $\mathrm{B}$ & 43 & 0 & 0 \\
\cline { 2 - 5 } & $\mathrm{C}$ & 62 & 4 & 6.45 \\
\cline { 2 - 5 } & $\mathrm{D}$ & 166 & 30 & 18.07 \\
\hline Pigeon & $\mathrm{A}$ & 51 & 3 & 5.88 \\
\cline { 2 - 5 } & $\mathrm{B}$ & 20 & 3 & 15 \\
\cline { 2 - 5 } & $\mathrm{C}$ & 119 & 3 & 2.52 \\
\cline { 2 - 5 } & $\mathrm{D}$ & 122 & 28 & 2.95 \\
\hline
\end{tabular}

reported in Jingzhou city (25.17\%) [10], but similar to that in Zhangjiakou city (7.41\%) [9], and Guangzhou city (8.43\%) [8]. The seroprevalence in caged chickens and free-range chickens was $6.23 \%$ and $10.19 \%$, respectively, although the difference was not statistically significant $(P>0.05)$.

The overall $T$. gondii seroprevalence in ducks in Lanzhou was $11.38 \%$, which was lower than that reported in other countries [16-18], and also lower than that reported in Guangzhou city (16\%) [8]. The differences in seroprevalence may due to differences in ecological and geographical factors. Of these, the T. gondii seroprevalence in caged ducks and free-range ducks were $6.31 \%$ and $13.90 \%$, respectively, but the difference was not statistically significant $(P>0.05)$, probably related to different life styles of the examined ducks.

To our knowledge, there was only one report regarding toxoplasmosis in pigeons in mainland China [14]. In the present study, antibodies to $T$. gondii were found in $37(11.86 \%)$ pigeons at the cut-off of $1: 5$, but most had low titres, with MAT titres of 1:5 in 35 pigeons, only 2 pigeons had titres of 1:10 or higher. This seroprevalence was higher than that in Guangdong Province (8.7\%) [14], possibly indicating geographical differences. Pigeon meat can serve as a source of $T$. gondii infection for hunters and other animals, so it would be a risk factor for T. gondii infection in humans or other animals.

In this study, we chose MAT because it is sensitive and specific for detecting $T$. gondii antibodies in bird species [2,8,13-15], compared to other serologic methods. Moreover, geographical conditions, feeding and living styles, and number of cats and rodents may contribute to the differences in $T$. gondii seropositivity in birds.

\section{Conclusions}

The present survey revealed the seroprevalence of T. gondii in chickens, ducks and pigeons in Lanzhou, northwest China for the first time, which indicated the potential risk of domestic birds as a source of $T$. gondii infection in humans and other animals in this region.

\section{Competing interests}

The authors declare that they have no competing interests.

\section{Acknowledgements}

This work was supported, in part, by the National Natural Science Foundation of China (Grant Nos. 31172316 and 31101812), the Program for Outstanding Scientists in Agricultural Research, the Open Funds of the State Key Laboratory of Veterinary Etiological Biology, Lanzhou Veterinary Research Institute, Chinese Academy of Agricultural Sciences (Grant Nos. SKLVEB2011KFKT004, SKLVEB2010KFKT009 and SKLVEB2011KFKT010) and the Yunnan Provincial Program for Introducing High-level Scientists (Grant No. 2009C1125). The authors thank Dr J. P. Dubey, Animal Parasitic Diseases Laboratory, Animal and Natural Resources Institute, Beltsville Agricultural Research Center, United States Department of Agriculture, USA for providing the Toxoplasma gondii MAT antigen.

\section{Author details}

'State Key Laboratory of Veterinary Etiological Biology, Key Laboratory of Veterinary Parasitology of Gansu Province, Lanzhou Veterinary Research Institute, Chinese Academy of Agricultural Sciences, Lanzhou, Gansu Province 730046, People's Republic of China. ${ }^{2}$ College of Animal Science and Technology, Jilin Agriculture University, Changchun, Jilin Province 130118, People's Republic of China. ${ }^{3}$ Department of Pathogen Biology and Immunology, Laboratory of Infection and Immunity, Xuzhou Medical College, Xuzhou, Jiangsu Province 221004, People's Republic of China. ${ }^{4}$ College of Animal Science and Technology, Yunnan Agricultural University, Kunming, Yunnan Province 650201, People's Republic of China.

\section{Authors' contributions}

$\mathrm{XQZ}$ conceived and designed the study, and critically revised the manuscript. WC, SYH, DHZ, MJX and SMW performed the experiments, analysed the data and drafted the manuscript. CY, QZ, and HQS helped in study design, study implementation and manuscript revision. All authors read and approved the final manuscript.

Received: 1 April 2012 Accepted: 7 June 2012

Published: 7 June 2012

\section{References}

1. Dubey JP: Toxoplasmosis of Animals and Humans. Secondth edition. Boca Raton, New York: CRC Press Inc; 2010:1-313.

2. Dubey JP: Toxoplasma gondii infections in chickens (Gallus domesticus): prevalence, clinical disease, diagnosis and public health significance. Zoonoses Public Health 2010, 57:60-73.

3. Zhou P, Chen Z, Li HL, Zheng H, He S, Lin RQ, Zhu XQ: Toxoplasma gondii infection in humans in China. Parasit Vectors 2011, 4:165.

4. Montoya JG, Liesenfeld O: Toxoplasmosis. Lancet 2004, 363:1965-1976.

5. Maksimov P, Buschtöns S, Herrmann DC, Conraths FJ, Görlich K, Tenter AM, Dubey JP, Nagel-Kohl U, Thoms B, Bötcher L, Kühne M, Schares G: Serological survey and risk factors for Toxoplasma gondii in domestic ducks and geese in Lower Saxony, Germany. Vet Parasitol 2011, 182:140-149.

6. Bártová E, Sedlák K, Literák I: Serologic survey for toxoplasmosis in domestic birds from the Czech Republic. Avian Pathol 2009, 38:317-320.

7. Bártová E, Dvoráková H, Bárta J, Sedlák K, Literák I: Susceptibility of the domestic duck (Anas platyrhynchos) to experimental infection with Toxoplasma gondii oocysts. Avian Pathol 2004, 33:153-157.

8. Yan C, Yue CL, Yuan ZG, He Y, Yin CC, Lin RQ, Dubey JP, Zhu XQ: Toxoplasma gondii infection in domestic ducks, free-range and caged chickens in southern China. Vet Parasitol 2009, 65:337-340. 
9. Tian PR, Cui P: Investigation on Toxoplasma gondii infection in chickens in Zhangjiakou city. Ani Husbandry Feed Sci 2010, 31:172-173. in Chinese.

10. Jiang $T$, Yin $C B$, Ruan $Z H$, He HS: Investigation on Toxoplasma gondii infection in chickens in Jingzhou city. Poultry Husbandry Dis Control 2010, 2:38-39. in Chinese.

11. Wu SM, Huang SY, Fu BQ, Liu GY, Chen JX, Chen MX, Yuan ZG, Zhou DH, Weng YB, Zhu XQ, Ye DH: Seroprevalence of Toxoplasma gondii infection in pet dogs in Lanzhou, Northwest China. Parasit Vectors 2011, 4:64.

12. Wu SM, Zhu XQ, Zhou DH, Fu BQ, Chen J, Yang JF, Song HQ, Weng YB, Ye DH: Seroprevalence of Toxoplasma gondii infection in household and stray cats in Lanzhou, northwest China. Parasit Vectors 2011, 4:214.

13. Dubey JP, Ruff MD, Camargo ME, Shen SK, Wilkins GL, Kwok OCH, Thulliez P. Serologic and parasitologic responses of domestic chickens after oral inoculation with Toxoplasma gondii oocysts. Am J Vet Res 1993, 54:1668-1672

14. Yan C, Yue CL, Qiu SB, Li HL, Zhang H, Song HQ, Huang SY, Zou FC, Liao M, Zhu XQ: Seroprevalence of Toxoplasma gondii infection in domestic pigeon (Columba livia) in Guangdong Province of southern China. Vet Parasitol 2011, 177:371-373.

15. Yan C, Yue CL, Zhang H, Yin CC, He Y, Yuan ZG, Lin RQ, Song HQ, Zhang KX, Zhu XQ: Serological survey of Toxoplasma gondii infection in the domestic goose (Anser domestica) in southern China. Zoonoses Public Health 2011, 58:299-302.

16. El-Massry A, Mahdy OA, El-Ghaysh A, Dubey JP: Prevalence of Toxoplasma gondii antibodies in sera of turkeys, chickens, and ducks from Egypt. J Parasitol 2000, 86:627-628.

17. Sroka J, Wojcik-Fatla A, Szymanska J, Dutkiewicz J, Zajac V, Zwolinski J: The occurrence of Toxoplasma gondii infection in people and animal from rural environment of Lublin region estimate of potential role of water a source of infection. Ann Agric Environ Med 2010, 17:125-132.

18. Murao T, Omata Y, Kano R, Murata S, Okada T, Konnai S, Asakawa M, Ohashi K, Onuma M: Serological survey of Toxoplasma gondii in wild waterfowl in Chukotka, Kamchatka, Russia and Hokkaido, Japan. J Parasitol 2008, 94:830833.

doi:10.1186/1756-3305-5-110

Cite this article as: Cong et al:: First report of Toxoplasma gondii

infection in market-sold adult chickens, ducks and pigeons in northwest China. Parasites \& Vectors 2012 5:110.

\section{Submit your next manuscript to BioMed Central and take full advantage of:}

- Convenient online submission

- Thorough peer review

- No space constraints or color figure charges

- Immediate publication on acceptance

- Inclusion in PubMed, CAS, Scopus and Google Scholar

- Research which is freely available for redistribution 